\title{
Multi-Object Tracking with Deep Learning Ensemble for Unmanned Aerial System Applications
}

\author{
Wanlin Xie ${ }^{\mathrm{a}}$, Jaime Ide ${ }^{\mathrm{a}}$, Daniel Izadi ${ }^{\mathrm{a}}$, Sean Banger ${ }^{\mathrm{a}}$, Thayne Walker ${ }^{\mathrm{a}}$, Ryan Ceresani ${ }^{\mathrm{a}}$, Dylan \\ Spagnuolo $^{\mathrm{a}}$, Christopher Guagliano ${ }^{\mathrm{a}}$, Henry Diaz ${ }^{\mathrm{a}}$, and Jason Twedt ${ }^{\mathrm{a}}$ \\ ${ }^{\mathrm{a}}$ Lockheed Martin AI Center
}

\begin{abstract}
Multi-object tracking (MOT) is a crucial component of situational awareness in military defense applications. With the growing use of unmanned aerial systems (UASs), MOT methods for aerial surveillance is in high demand. Application of MOT in UAS presents specific challenges such as moving sensor, changing zoom levels, dynamic background, illumination changes, obscurations and small objects. In this work, we present a robust object tracking architecture aimed to accommodate for the noise in real-time situations. We propose a kinematic prediction model, called Deep Extended Kalman Filter (DeepEKF), in which a sequence-to-sequence architecture is used to predict entity trajectories in latent space. DeepEKF utilizes a learned image embedding along with an attention mechanism trained to weight the importance of areas in an image to predict future states. For the visual scoring, we experiment with different similarity measures to calculate distance based on entity appearances, including a convolutional neural network (CNN) encoder, pre-trained using Siamese networks. In initial evaluation experiments, we show that our method, combining scoring structure of the kinematic and visual models within a MHT framework, has improved performance especially in edge cases where entity motion is unpredictable, or the data presents frames with significant gaps.
\end{abstract}

Keywords: multi-object tracking, deep neural network, extended kalman filter, long short-term memory network, multiple hypothesis tracking

\section{INTRODUCTION}

Unmanned aerial systems (UAS) are commonly used for intelligence surveillance and reconnaissance (ISR) missions to provide critical information to decision makers. Situational awareness (SA) is enhanced when up-to-date, coherent, and trustworthy information is disseminated to all areas of the battlefield. Artificial Intelligence and Machine Learning (AI/ML) deployed at the edge is a key enabler to achieving this. Edge compute devices provide the infrastructure for deep learning methods to accelerate information extraction from onboard sensors and disseminate over communication networks. A full understanding of the battlefield requires all entities and their intent be known and understood. Multi-object tracking (MOT) is a crucial component to achieving this. ${ }^{1-3}$ Application of MOT in UAS presents specific challenges such as moving platform and sensor, large variations in view point and pose, fast and abrupt object motion, changing zoom levels, illumination changes, occlusions, low resolution of detected objects due to small sizes. ${ }^{4,5}$

Further author information: (Send correspondence to J.T.)

J.T.: E-mail: jason.c.twedt@lmco.com 
Our solution is based on the tracking-by-detection paradigm. ${ }^{6-8}$ As shown in Figure 1, platform information, images and other sensor data are received by the multi-object tracker (MOT). Objects are identified as Detections by the Object Detector. A Detection consists of a bounding box on each object entity as well as the corresponding entity label. Detections are then sent to the Object Tracker, which is composed of an Associator and Similarity Fuser. The job of the Associator is to link together detections between frames to create object trajectories. The Similarity fuser is a modular scoring component that compiles all the scores from the Signature Comparators into a final metric.

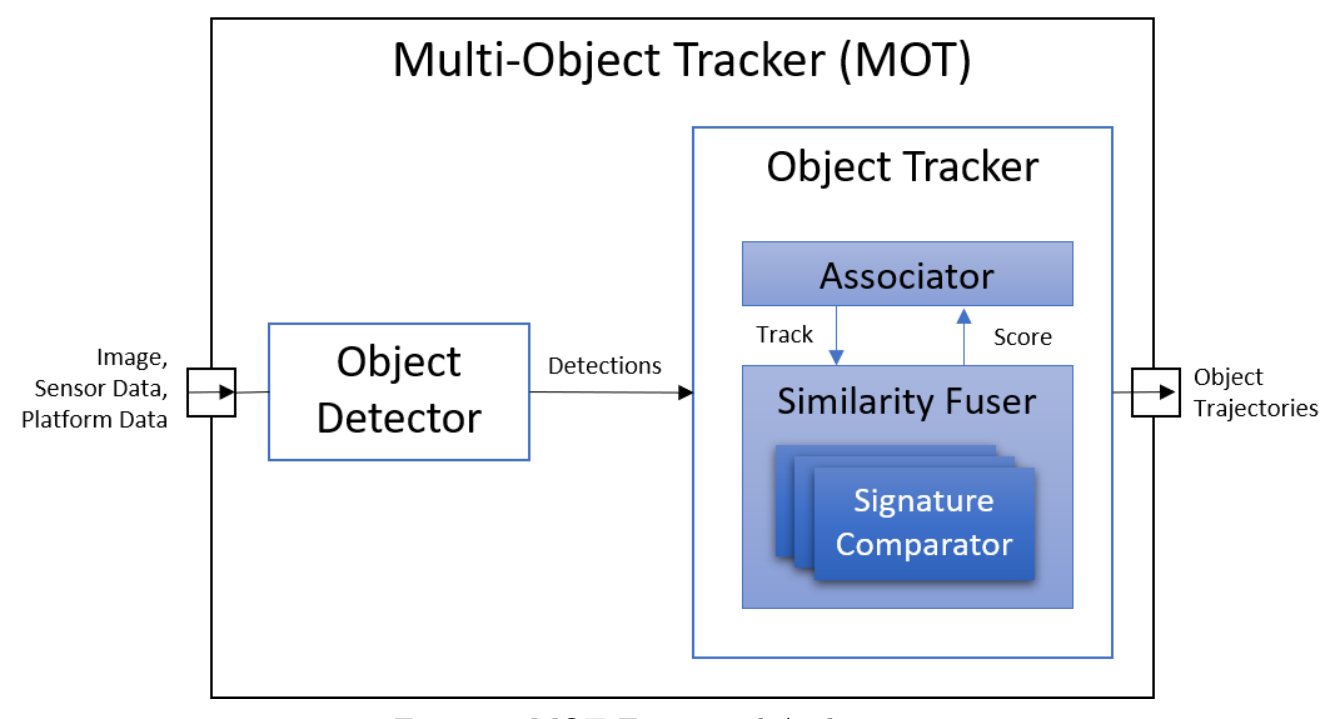

Figure 1. MOT Functional Architecture

Track performance is highly dependent on the similarity computation between detections and/or tracklets with both motion and appearance methods playing a significant role. ${ }^{9}$ To this end, we propose an architecture that is composed of an ensemble of Signature Comparators, each targeting different aspects of tracking. We broadly separate these Signature Comparators into two categories, short-term and long-term. In both cases, we aim to exploit the uniqueness of an object through detection of intrinsic signatures (fingerprint) using deep learning. short-term methods focus on modeling the motion of objects in subsequent frames over short durations. Here an object's anticipated motion or kinematic signature is exploited to associate the object in future frames. Over periods of long occlusions or as objects move in and out of the sensor field-of-view (FOV), motion based models can become less accurate due to error propagation over time. In these cases we exploit an object's visual signature for re-identification of the object in future frames (long-term tracking). The output of all Signature Comparators are combined into a single score by the Similarity Fuser and provided to the Associator for track ID association. In this work, we combine recent advances in deep learning ${ }^{10,11}$ with traditional object tracking methods. ${ }^{12,13}$

We implement the Associator as a tree-based multiple hypothesis tracking (MHT) ${ }^{14,15}$ framework that produces the best hypothesis based on the kinematic and visual information extracted from the detections. MHT provides a robust tracking system to deal with misdetections and occlusions by keeping multiple trees of plausible object trajectories. However, it heavily relies on the accuracy of the kinematic and visual scores. Therefore, we propose improvements in two folds through the addition of both short and long-term Signature Comparators:

- Deep learning the kinematic scores. In MHT, high kinematic scores are given to objects that are observed close to their predictions. Thus, the accuracy of the kinematic model will drive the reliability of the kinematic scores. Traditionally, this motion prediction can be done using a standard Kalman Filter. ${ }^{16}$ This works well on a simulated scene or when a camera is fixed at a stationary position, but is unreliable as more real world factors are introduced, e.g. non-linear motion of the aircraft, sensor pointing angles, sensor zoom, and object trajectories. In this work, we present a novel model using deep learning models 
integrated within an extended Kalman Filter $(\mathrm{EKF})^{17}$ based Signature Comparator to predict nonlinear and complex movement patterns. We propose a kinematic prediction model, called DeepEKF, in which we use a sequence-to-sequence architecture to predict entity trajectories in latent space. DeepEKF utilizes a learned image embedding along with an attention mechanism that is trained to weight the importance of areas in an image to predict future states. The model is composed of a dual deep neural network (DNN) ${ }^{18}$ - a kinematic prediction model with an Encoder-Decoder Long Short-Term Memory (LSTM) architecture trained end-to-end with a convolutional neural network $(\mathrm{CNN})^{19}$

- Deep learning the visual scores. Along with the kinematic score, the visual similarity between two detected objects is used to estimate the final score of the tracking hypothesis in MHT. We employ the sum square difference (SSD) ${ }^{15}$ as the baseline measure, and propose a deep learning model to estimate the similarity score between two image chips. The model uses a CNN architecture and it is trained to learn image embeddings that are optimal in identifying unique entities. The training is done using Siamese networks. ${ }^{20,21}$ Our Siamese network architecture is an multi-headed attention-driven model that is able to focus on specific spatial regions of interest to create a unique object fingerprint. This fingerprint may be used to perform visual object re-identification in the absence of reliable kinematic data.

In initial evaluation experiments, we show that our method, combining scoring structure of the kinematic and visual models within a MHT framework, has improved performance especially in edge cases where entity motion is unpredictable, or the data presents frames with significant gaps.

\section{RELATED WORK}

Motion based affinity models predict a future object state and then compare that state to received detections for similarity scoring. Kalman filters then predict future bounding box coordinates and generate an affinity score from intersection over union (IOU), ${ }^{22}$ and assumes linear motion. A purely visual approach proposed in $^{23}$ uses a visual displacement CNN that takes stacked image chips as input to predict an object's future location in a discretized frame. Recurrent neural networks (RNNs) have been proposed to model non-linear motion and measurement noise ${ }^{24}$ and to overcome the long-term occulsion problem. ${ }^{25}$ Approaches that use a pure visual approach or the IOU score of detections work well on a stationary camera where the next frame points to the same position, but becomes unreliable when utilized in a scenario with a moving camera.

Visual object re-identification is a rising area of research and much of the recent work has been improving re-identification using multiple image comparisons, ${ }^{26}$ utilizing attention, ${ }^{27}$ unsupervised learning, ${ }^{28}$ body part information fusion, ${ }^{29}$ and creating clothing agnostic models using generative networks. ${ }^{30,31}$ Many open source models are not designed in the context of long term re-identification (appearance agnostic) and fall short when challenged with appearance changes. Research that is geared towards appearance agnostic re-identification often times relies on either generative models or utilizing part detection. One approach to using generative models is to project an image into the context of another (pose, color, etc.) ${ }^{30}$ before performing image comparisons. This method has the potential to be memory and timing intensive. Other generative models utilize image reconstruction in a multi-task learning context to enforce that the feature extractor learns to encode body structure ${ }^{31}$ before globally pooling the features. Outside of generative models, utilizing part detection ${ }^{29}$ guides the visual comparison network to focus on specific regions of interest in the image that may be generally appearance agnostic. The part detection method removes the ability for the model to identify more significant regions of interest and is not guaranteed to be robust against appearance changes within the detected parts. In contrast, our visual re-identification module uses a spatial attention head to identify important and unique areas of interest in an image with a method that avoids global pooling. Our method supports the use of single and multiple image comparisons and is adaptable to different viewing angles. Our approach also does not preclude the inclusion of additional multi-task loss functions that may prove effective for remaining appearance agnostic.

Work in integrating deep learning techniques in the multiple hypothesis tracking (MHT) framework is growing. Kim et al. ${ }^{32}$ used LSTM networks to model object appearance over time, utilizing a vanilla LSTM to process motion data and bilinear LSTM to process appearance data. $\mathrm{CNNs}^{33}$ have also been used in MHT where the CNN was used for feature extraction and combined with a Kalman Filter for motion prediction. Our approach 
offers the flexibility to combine multiple affinity models in MHT while most approaches have only looked at using a single network. We also introduce a novel deep learning network focused on motion prediction to inform a kinematic likelihood during the MHT gating step.

\section{BACKGROUND}

\subsection{State Estimation}

The Kalman filter (KF) is a popular algorithm for target state estimation from noisy observations. It has various applications including autonomous vehicles or stock market prediction. The KF models transition to the next state as a linear function of the current state. Kalman filters are composed of a two-step iterative process including a prediction step and an update step that estimates the mean and variance of a Gaussian distribution. The Extended Kalman Filter (EKF) models nonlinear state transitions and/or measurement models that are nonlinear functions of state. The likelihood that a measurement is generated from a Kalman filter state is given by the multivariate probability density function. ${ }^{34}$

An MOT application deployed for aerial ISR missions, must be robust to diverse operational environments and target sets. The aircraft, sensor pointing angles, sensor zoom, and targets of interest exhibit complex (nonlinear) behavior that cannot be specified a-priori. A solution that dynamically adapts to changing state parameters in aircraft, sensor and targets is desired. Deep learning has shown to be effective in this respect. Learned extensions to Kalman filters with LSTMs have been shown to improve estimation accuracy. ${ }^{35,36}$ When applied to pixelbased tracking, LSTMs show effectiveness in learning the motion of generic objects based on appearance. ${ }^{37}$ Our approach builds upon prior LSTM motion model architectures that leverage appearance features and augments them to include system parameters such as aircraft and sensor state. The network architecture is modeled after an EKF and includes state prediction, noise estimation and likelihood calculation within an N-dimensional latent space.

\subsection{General Visual Comparison Methods}

Current visual object tracking models use the method of feature learning, which creates a feature vector representation of the object being tracked. These models are trained on ranking loss functions with the objective of minimizing the agreement between features of dissimilar labels and maximizing agreement between features of the same label in latent space. Typical metrics for inference of visual feature embeddings are Cosine Similarity and Euclidean Distances between two embeddings to create a similarity score for a comparison between two images. These scores can either be ranked against a gallery of embeddings to find the most likely match, or paired with a margin to establish whether or not a pair of images is a match.

Many existing models use popular CNN models such as ResNet, ${ }^{38}$ EfficientNet, ${ }^{39}$ GoogleNet, ${ }^{40}$ etc. for the backbone of their re-identification models. For the purpose of visual feature learning for tracking, there are three typical approaches to training these models. The first is to use a global feature from the entire image, and train on classification loss. The second is to use a ranking loss function to maximize the score between global features of similar objects. The last is to learn part-based features rather than global features. Most state-of-the-art (SOTA) models use a combination of a ranking loss function on both global and local features, and classification loss on the unique ids in the training set improve generalizability and performance of their models. ${ }^{28,41}$

\subsection{Multiple Hypothesis Tracking}

Data association is at the core of multi-object tracking (MOT), and since its creation, multiple hypothesis tracking (MHT) ${ }^{14}$ became the standard method for MOT when data association is challenging due to occlusion, low probability of detection or high target density. ${ }^{13,15,42-46}$ A computationally more efficient alternative to the original hypothesis-oriented MHT has been proposed and it is known as the tracking-oriented MHT. ${ }^{44,47}$ As opposed to the original MHT in which multiple hypotheses are expanded and kept across frames, in trackoriented MHT, association hypotheses are maintained at the individual track level. Thus, hypotheses with low probability or that are contradictory are deleted, and most likely hypothesis tracks are kept.

With the advent of efficient and accurate object detection algorithms provided by deep learning methods, MHT approaches using the tracking-by-detection framework are popular. ${ }^{15}$ In this approach, objects along with 
their bounding boxes are automatically generated, and used as the observations within MHT. A track hypothesis is defined as a sequence of observations across frames with the same unique identifier. The global hypothesis is defined as the set of track hypotheses that are not in conflict. The key idea in MHT is to create multiple track trees representing unique objects, and keep them active across frames, until association ambiguities are resolved. At each frame, track trees are updated from the observations, scored, and the best set of non-conflicting tracks (i.e., best global hypothesis) can be found solving a maximum weighted independent set (MWIS) problem. ${ }^{48}$ Multi-hypothesis tracking makes up the framework of the object tracker, storing hypothesis tracks in a tree-based structure. Each tree in the framework represents a single tracked entity. Each branch in the tree represents a hypothetical trajectory of the given object entity - each node a tracked detection at a specific timestamp.

\section{METHODS}

\subsection{Short-Term Tracking}

In this work, we use vision to enhance kinematic projections for improved state estimation and uncertainty estimation while utilizing the objects' surroundings and own appearance to predict future motion. This approach combines both visual and kinematic aspects of aerial ISR. The dynamics of the aircraft motion, sensor pointing, optical zoom, and target motion are modeled in an encoder-decoder paradigm.

We utilize an attention mechanism to allow the decoder to selectively attend to regions in the scene most important to each projection timestep. Objects within the scene may influence the target's behavior at different times e.g., stationary, or non-stationary obstacles.

DeepEKF determines the similarity between a tracklet and detection (i.e., measurement) by computing the likelihood from the DeepEKF filter state. First the target tracklet is encoded via the RNN Encoder, then decoded with the RNN Decoder with Attention. The output is a $n$-dimensional projection of the target object in latent space with a position and uncertainty ellipse at $t_{N}+\Delta t$. All measurements are then passed through the same RNN Encoder and Decoder (same weights), with the only difference being the RNN Encoder's hidden state is initialized from the output of the target encoding pass. The result is that each measurement will have a state and covariance that can be compared to the target projection. The residual position and covariance are used to compute the likelihood value. For the 2D case, the more overlap between ellipses, the greater the likelihood that the tracklet produced the measurement (Figure 2).
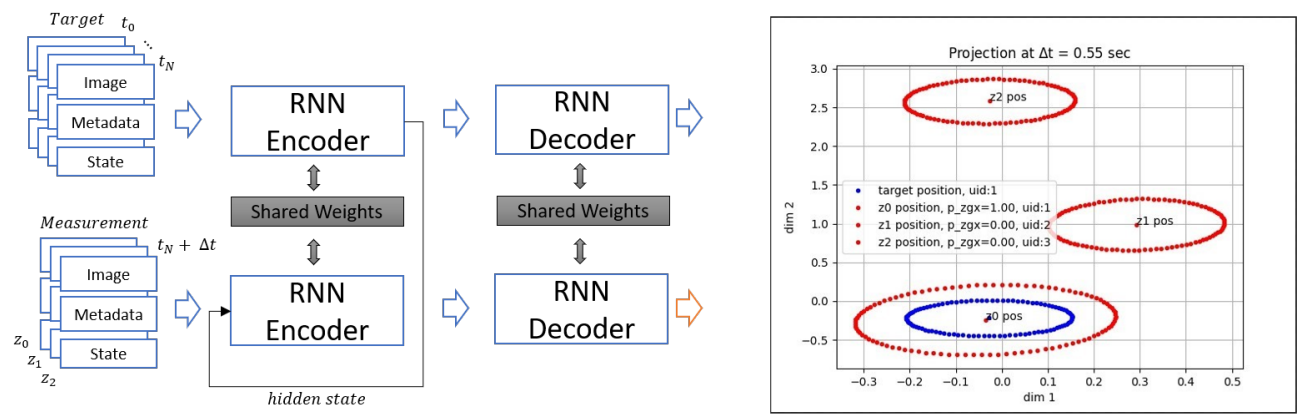

Figure 2. DeepEKF affinity example of score in the 2D latent space.

\subsection{Long-Term Tracking (Re-identification) with Siamese Networks}

Kinematic data is not always reliable for object tracking particularly in occluded scenes for objects that do not have well-defined kinematic behavior. In these situations visual identification is important to ensure that multiple tracks are not formed on a single object. Siamese networks are important for visual identification because they are able to learn a unique latent space embedding of an object using visual features that can be used for re-identification. Context plays an important role in re-identification so it is important to note the difference between short-term re-identification and long-term re-identification. Short-term re-identification in the context of object tracking generally realizes lower object appearance variance when compared to long-term re-identification. 
It is due to this increased variance for long-term re-identification that makes it challenging to perform in the real-world; particularly when paired with potentially low resolution data from an aerial perspective. One such issue is clothing changes between detections for person re-identification since existing open-source models often times identify features that would fail under these circumstances.

Our Siamese network architecture $\mathrm{e}^{20,21}$ is an attention-driven model that is able to focus on specific object features of interest to create its latent space embedding. Our modular architecture consists of a four basic components known as the backbone, neck, decoder, and head. The backbone extracts visual feature maps from the image using a feature extractor network such as a convolutional neural network (CNN) or vision transformers (ViT). The neck acts as a translation mechanism that embeds the feature maps from the backbone into a format suitable for the decoder. The decoder globally attends to the feature maps to suppress irrelevant spatial locations in the image. Finally, the head is an attention model that takes the visual feature embedding and identifies spatial areas of interest that are used to form the multiple final latent space embeddings.

Our models were evaluated on the open source Market-1501 dataset ${ }^{49}$ with the official testing protocol that uses a set of query images to find the correct match across a set of gallery of images. Our attention-driven Siamese network model was able to achieve 99.4\% Rank-1 accuracy and $74.9 \%$ mAP using this evaluation protocol. Example attention maps for each attention head can be found in Figure 3.

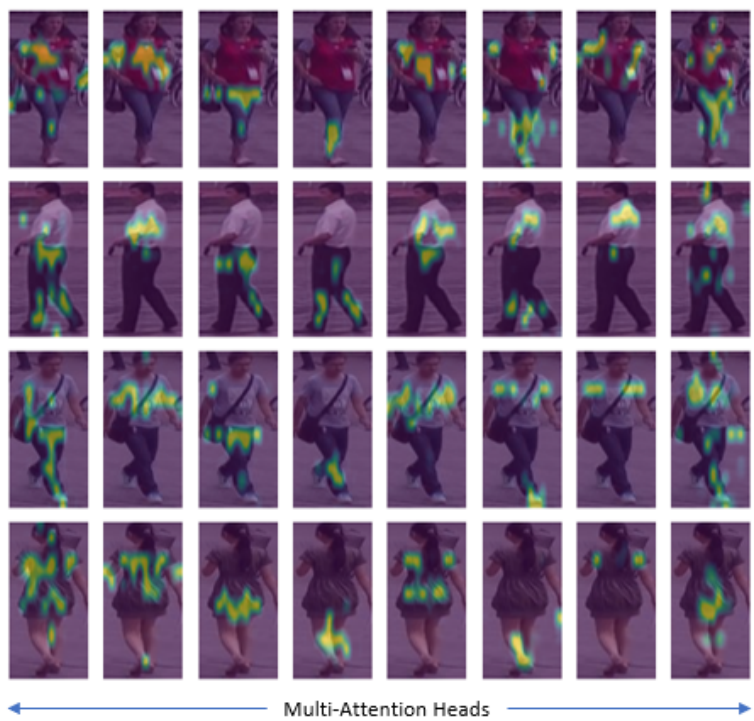

Figure 3. Attention heads for example Market-1501 images

We also trained and tested a similar Siamese network architecture, without an attention mechanism. This Siamese network architecture passes the image through a CNN backbone directly into an MLP head. This model was also evaluated on the Market-1501 dataset, and was able to achieve $90.7 \%$ Rank-1 accuracy and and $70.1 \%$ mAP using single image comparisons. In Figure 4, we can visualize a t-SNE plot of the features extracted using the trained Siamese network on a sample dataset including images of ten distinct detections ( 7 people and 3 vehicles).

\section{EXPERIMENTS}

\subsection{Data}

The data was collected from numerous flights of a fixed-wing UAS with gimbaled EO camera. The collected video was labeled using a combination of in-house and external labeling tools. We run experiments on the following two video sequences of different scenarios involving object entities of people and vehicle, described in Table 1. In Figures 5 and 6, we illustrate some frames from the dataset. All images have the original size. In Video 1 


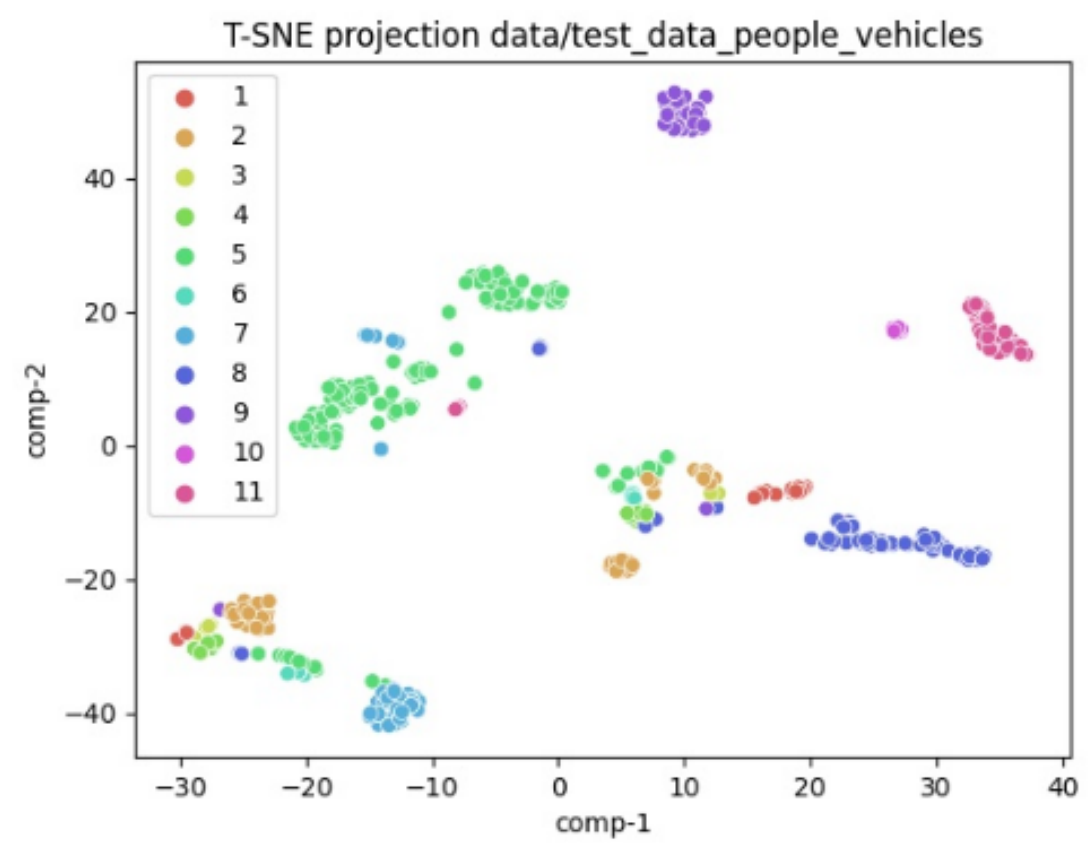

Figure 4. T-SNE plot for test Video 1. The output embeddings of the Siamese network model are clustered in latent space by an unique identifier (UID).

(Figure 5), we have three people walking through a field with significant changes in scale, point of view, pose and color, with two of them disappearing and reappearing in the scene. In Video 2 (Figure 6), we have two people running across a road. It presents significant changes in scale, and point of view, with both runners disappearing and reappearing in the scene twice along with the camera zooming in. These datasets are challenging from both kinematic and visual modeling perspectives. It has abrupt changes in positioning and relatively long periods of absence from the scene (10 frames), as well as objects with very similar appearances.

Table 1. Dataset description.

\begin{tabular}{|l|l|l|l|}
\hline Video & Description & Frame Length & Kinematic Metadata \\
\hline 1 & three people walking in field & 124 & True \\
\hline 2 & two people running alongside a road & 313 & True \\
\hline
\end{tabular}

We compile together multiple trajectories for each object entity in a video sequence to train our model's kinematic prediction output. The dataloader reads in image and metadata files and automatically generates sequences of varying input and output sequence length. Each frame in the sequence contains the full object's characteristics including kinematic metadata (e.g. longitude, latitude, camera position).

\subsection{Performance Measures}

Unlike widely accepted definitions for precision and recall in the case of classification problems, defining performance measures for object tracking is more ambiguous. We want to precisely diagnose how well our model can accurately and consistently keep track of distinct object entities over continuous periods of time.

Our ground truth and predicted data come in a frame-by-frame format containing detection information bounding box, feature characteristics, and tracking id - as well as kinematic metadata informing the sensor and geographic coordinate information. Unlike bounding box coordinates and feature characteristics, ground truth 

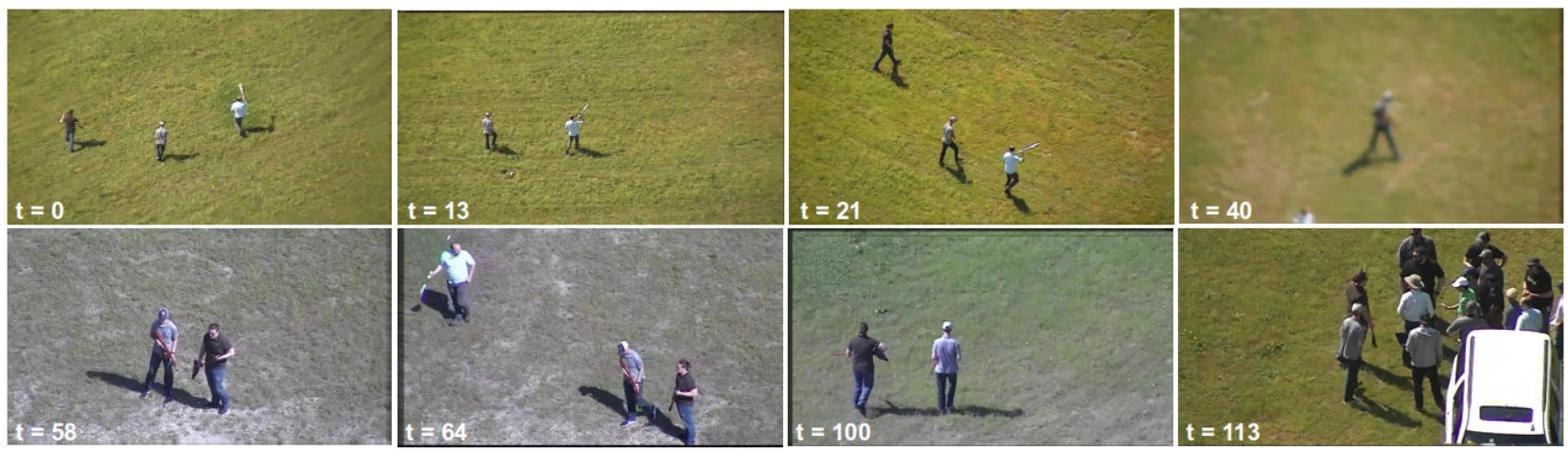

Figure 5. Sample frames from Video 1. Three people walking on an open field. Frame 0: initial frame showing the 3 people. Frame 13: person on the left with black t-shirt walked out of the scene. Frame 21: person with black t-shirt is back. Frame 40: blurred image. Frame 58: notice the changes in illumination and color. Frame 64: third person with white t-shirt is back. Frame 100: changes in point of view. Frame 113: two people join a group.
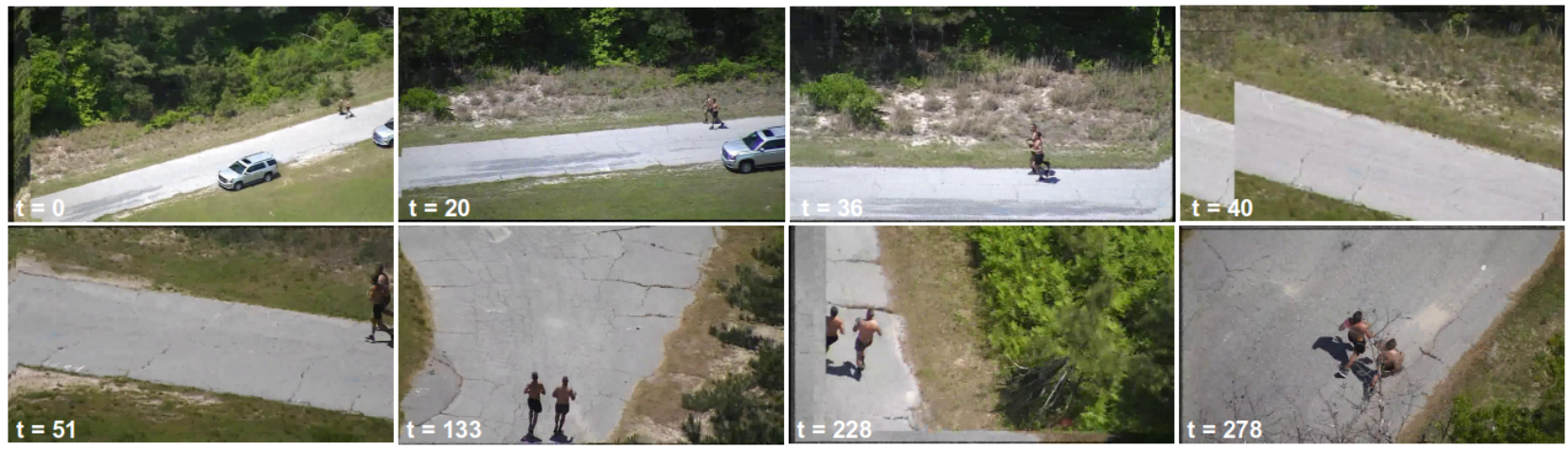

Figure 6. Sample frames from Video 2. Two people running across a road. Frame 0: initial frame showing the 2 runners. Frame 20: notice the small size of the entities. Frame 36: second runner is partially occluded and overlapping with the other runner. Frame 40: runners are out of the scene. Frame 51: runners are back after a gap of 10 frames. Frame 133: from far, the two runners are identical. Frame 228: camera movements are abrupt and cause distortions in the image. Frame 278: significant change in point of view.

tracking id labels will most often not match predicted id labels from the object tracker, so we must add an additional layer to determine the optimal mapping of tracking ids.

We look at several performance measures including absence prediction, precision recall plots, longevity of tracking, etc. The primary metric we utilize to benchmark our object tracking experiments is expected average overlap (EAO), introduced in the Visual Object Tracking (VOT) challenge, ${ }^{50}$ an averaged recall score that is measured between a range of sequence lengths defined by a kernel density estimate.

\subsection{Results and Discussion}

For each video, given the pre-computed object detections along with their bounding boxes, we applied different OT model architectures, and computed the EAO looking at consistency with the original associated track ID as well as newly created track IDs. The original image bounding boxes were resized to $(100,100)$, and the visual components (SSD and SiameseNet models) were computed. Results are shown in Tables 2 and 3. Follow the description of the used models:

- Greedy: EKF (non-NN). Tracking performed using standard EKF, only based on the kinematic information of the detected objects. Pixel coordinates are used and there is no neural networks involved. 
- MHT: EKF(pixel-based) + SSD. Kinematic score is computed using EKF estimates of pixel coordinates. Visual score is directly computed from the sum squared differences of the detected objects. $50 \% / 50 \%$ weights are given to the kinematic and visual components.

- MHT: EKF(pixel-based) + SiameseNet. Kinematic score is computed using EKF estimates of pixel coordinates. The visual score is estimated by computing the Euclidean distance between the embedding generated using the Siamese networks. 50\%/50\% weights are given to the kinematic and visual components.

- MHT: EKF(pixel-based) + SiameseNet Attn. Kinematic score is computed using EKF estimates of pixel coordinates. The visual score is estimated by computing the Euclidean distance between the embedding generated using the Siamese networks with attention mechanism. 50\%/50\% weights are given to the kinematic and visual components.

- MHT: DEKF + SSD. Kinematic score is computed using trained DeepEKF model that estimates using visual and kinematic embeddings. Visual score is directly computed from the sum squared differences of the detected objects. 50\%/50\% weights are given to the kinematic and visual components.

- MHT: DEKF + SiameseNet. Kinematic score is computed using trained DeepEKF model that estimates using visual and kinematic embeddings. The visual score is estimated by computing the Euclidean distance between the embedding generated using the Siamese networks. 50\%/50\% weights are given to the kinematic and visual components.

- MHT: DEKF + SiameseNet Attn. Kinematic score is computed using trained DeepEKF model that estimates using visual and kinematic embeddings. The visual score is estimated by computing the Euclidean distance between the embedding generated using the Siamese networks with attention mechanism. $50 \% / 50 \%$ weights are given to the kinematic and visual components.

\begin{tabular}{|l|c|}
\hline Model & EAO (oUID) \\
& EAO (aUID) \\
\hline \multirow{2}{*}{ Greedy [EKF (non-NN)] } & $\mathbf{0 . 1 7 5 0}$ \\
& 0.6156 \\
\hline \multirow{2}{*}{ MHT [EKF (pixel-based), SSD] } & $\mathbf{0 . 0 2 0 8}$ \\
& 0.8839 \\
\hline \multirow{2}{*}{ MHT [EKF (pixel-based), SiameseNet] } & $\mathbf{0 . 1 9 5 8}$ \\
& 0.7497 \\
\hline \multirow{2}{*}{ MHT [EKF (pixel-based), SiameseNet Attn] } & $\mathbf{0 . 1 9 5 8}$ \\
& 0.7497 \\
\hline \multirow{2}{*}{ MHT [DEKF, SSD] } & $\mathbf{0 . 2 8 8 1}$ \\
& 0.7297 \\
\hline \multirow{2}{*}{ MHT [DEKF, SiameseNet] } & $\mathbf{0 . 2 2 5 6}$ \\
& 0.4861 \\
\hline \multirow{2}{*}{ MHT [DEKF, SiameseNet Attn] } & $\mathbf{0 . 4 4 6 4}$ \\
& 0.5922 \\
\hline
\end{tabular}

Table 2. Experiments on Video 1. EAO original UID (oUID) only gives credit to the predicted track ID that was first associated with a ground truth track id. EAO any UID (aUID) gives credit to multiple associated predicted track IDs as long as there is no disagreement.

In Table 2, the best EAO is 0.4464 for the DeepEKF using the Siamese with attention networks, followed by the DeepEKF with the standard SSD, EAO 0.2881. In this video, we have three people walking through a field with significant changes in scale, point of view, pose and color, with two of them disappearing and reappearing in the scene (as illustrated in Figure 5). 


\begin{tabular}{|l|c|}
\hline Model & EAO (oUID) \\
& EAO (aUID) \\
\hline \multirow{2}{*}{ Greedy [EKF (non-NN)] } & $\mathbf{0 . 0 2 2 1}$ \\
& 0.5897 \\
\hline \multirow{2}{*}{ MHT [EKF (pixel-based), SSD] } & $\mathbf{0 . 0 2 7 7}$ \\
& 0.8684 \\
\hline \multirow{2}{*}{ MHT [EKF (pixel-based), SiameseNet] } & $\mathbf{0 . 0 3 7 1}$ \\
& 0.6820 \\
\hline \multirow{2}{*}{ MHT [EKF (pixel-based), SiameseNet Attn] } & $\mathbf{0 . 0 3 7 1}$ \\
& 0.6694 \\
\hline \multirow{2}{*}{ MHT [DEKF, SSD] } & $\mathbf{0 . 1 4 9 4}$ \\
& 0.7586 \\
\hline \multirow{2}{*}{ MHT [DEKF, SiameseNet] } & $\mathbf{0 . 1 7 9 0}$ \\
& 0.2460 \\
\hline \multirow{2}{*}{ MHT [DEKF, SiameseNet Attn] } & $\mathbf{0 . 5 0 4 5}$ \\
& 0.5414 \\
\hline
\end{tabular}

Table 3. Experiments on Video 2.

In Table 3, the best EAO is 0.5045 for the DeepEKF using the Siamese with attention networks as well, followed by the DeepEKF with the standard Siamese network, EAO 0.1790. In this video, we have two people running across a road, and it presents significant changes in scale, and point of view, with both runners disappearing and reappearing in the scene twice (sample frames are illustrated in Figure 6). This dataset is challenging for both kinematic and visual modelings. It has abrupt changes in positioning and relatively long periods of absence from the scene (8-9 frames). Additionally, the two runners are very similar in appearance and only distinguishable using the positional information.

In both experiments, our proposed DeepEKF method, using the Siamese networks with attention to generate the visual scores, outperforms the other variants. This is likely because its ability to model non-linear kinematics and re-identify objects with significant changes in pose and view.
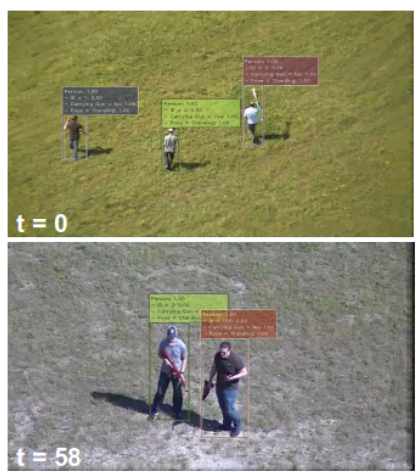
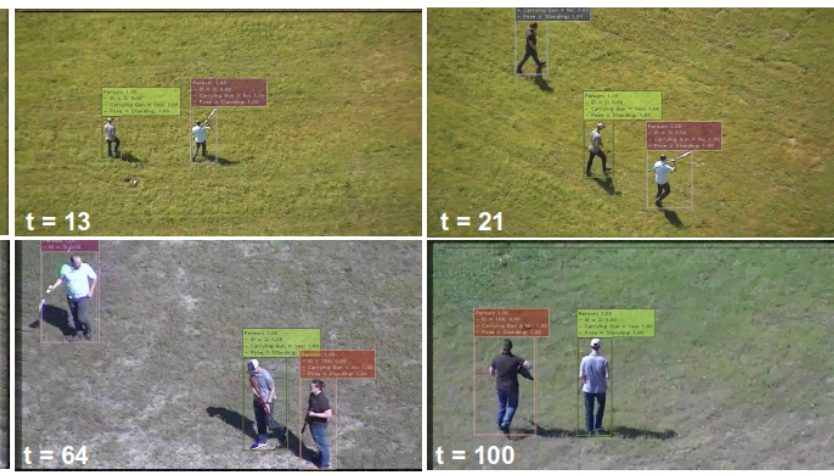

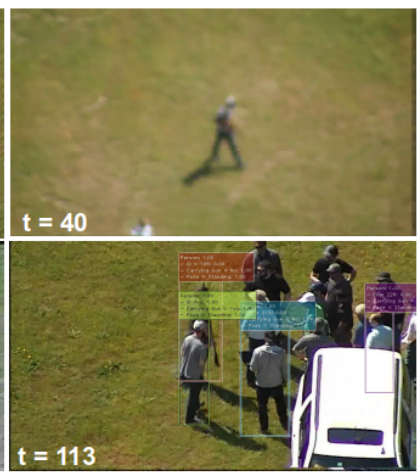

Figure 7. Tracking results from Video 1: Three people walking on an open field. Frame 0: initial tracking with 3 people. Unique identifiers (UIDs) are reported in the label box next to the identified object. Each UID is represented with different color. Frame 13: two UIDs are correctly identified. Frame 21: person with black t-shirt is back and correctly identified. Frame 40: tracking ignores poor quality detection. Frame 58: person with hat is correctly identified however a new ID is mistakenly assigned to the person with black t-shirt. Frame 64: third person with white t-shirt is back and correctly identified. Frame 100: person with black t-shirt is correctly tracked after frame 58. Frame 113: two persons continue being correctly tracked after joining a group.

We use the EAO (original UID) score as our primary metric (reported in Tables 2 and 3). The EAO (any UID) score does not penalize creating new track IDs for object entities, even if they have been seen before. 

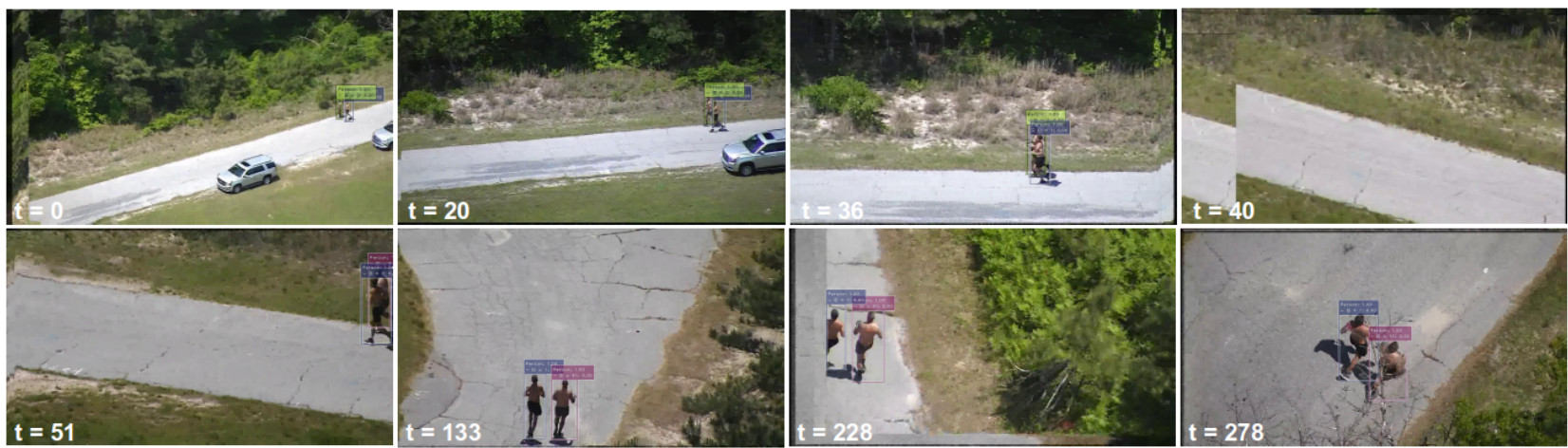

Figure 8. Tracking results from Video 2: Two people running across a road. Frame 0: initial frame showing the 2 runners. Unique identifiers (UIDs) are reported in the label box next to the identified object. Each UID is represented with different color. Frame 20: tracked objects are very close each other. Vehicle is ignored in this tracking experiment. Frame 36: runners are correctly identified even with overlapping objects. Frame 40: there is no detection but hypothesis trees are kept by the system. Frame 51: after a gap of 10 frames, the tracker correctly re-identifies the first but not the second runner. Frame 133: after the mistake at frame 51, the second runner continues being correctly tracked. Frame 228: first runner correctly tracked even with partial occlusion. Frame 278: both runners are correctly tracked until the end after frame 51 .

Therefore, a model can get a high score merely by assigning a new track ID every time. However, when the EAO (original UID) score is tied, the EAO (any UID) score is useful to determine which model will make less mistakes in assigning a current UID to the wrong object entity.

\section{FUTURE WORK}

In this paper, we proposed a DeepEKF architecture for MOT in UAS applications. The method was validated on two data sets, showing improvements of the DeepEKF over the standard KF within the MHT framework. Although we report a proof of concept results, further training of the DeepEKF as well as of the Siamese networks are necessary as we collect more data. In particular, we plan to add a more extensive evaluation for the longterm tracking (re-identification) component. Another promising venue is to dynamically combine the different kinematic and visual scores within the Similarity Fuser component (Figure 1) given the environment and track states.

\section{ACKNOWLEDGMENTS}

We thank David Rosenbluth for providing review and feedback on this work. We thank Andrew Walsh and Kenneth Rapko Jr. for their valuable feedback on benchmarking techniques in object tracking.

\section{REFERENCES}

[1] Asnis, G. and Blackman, S., "Optimal allocation of multi-platform sensor resources for multiple target tracking," in [14th International Conference on Information Fusion], 1-8 (2011).

[2] Blackman, S. S., Tyler, D., and Pickle, C. D., "Application of mht to through the wall radar tracking," in [2013 IEEE Aerospace Conference], 1-9 (2013).

[3] Shi, X., Taheri, A., Çeçen, T., and Çelik, N., "Particle filtering-based low-elevation target tracking with multipath interference over the ocean surface," IEEE Transactions on Aerospace and Electronic Systems 56(4), 3044-3054 (2020).

[4] Li, S. and Yeung, D.-Y., "Visual object tracking for unmanned aerial vehicles: A benchmark and new motion models," Proceedings of the AAAI Conference on Artificial Intelligence 31 (Feb. 2017). 
[5] Du, D., Qi, Y., Yu, H., Yang, Y., Duan, K., Li, G., Zhang, W., Huang, Q., and Tian, Q., "The unmanned aerial vehicle benchmark: Object detection and tracking," in [Computer Vision - ECCV 2018], Ferrari, V., Hebert, M., Sminchisescu, C., and Weiss, Y., eds., 375-391, Springer International Publishing, Cham (2018).

[6] Andriluka, M., Roth, S., and Schiele, B., "People-tracking-by-detection and people-detection-by-tracking," in [2008 IEEE Conference on Computer Vision and Pattern Recognition], 1-8 (2008).

[7] Porikli, F. and Yilmaz, A., [Object Detection and Tracking], 3-41, Springer Berlin Heidelberg, Berlin, Heidelberg (2012).

[8] Feichtenhofer, C., Pinz, A., and Zisserman, A., "Detect to track and track to detect," in [IEEE International Conference on Computer Vision (ICCV), 2017], (Oct. 2017).

[9] Ciaparrone, G., Luque Sánchez, F., Tabik, S., Troiano, L., Tagliaferri, R., and Herrera, F., "Deep learning in video multi-object tracking: A survey," Neurocomputing 381, 61-88 (Mar 2020).

[10] Zhao, Z.-Q., Zheng, P., Xu, S.-T., and Wu, X., "Object detection with deep learning: A review," IEEE Transactions on Neural Networks and Learning Systems 30(11), 3212-3232 (2019).

[11] Xu, Y., ŝep, A., Ban, Y., Horaud, R., Leal-Taixé, L., and Alameda-Pineda, X., "How to train your deep multi-object tracker," in [2020 IEEE/CVF Conference on Computer Vision and Pattern Recognition (CVPR)], 6786-6795 (2020).

[12] Pylyshyn, Z. and Storm, R., "Tracking multiple independent targets: evidence for a parallel tracking mechanism.," Spatial vision 3 3, 179-97 (1988).

[13] Chong, C.-Y., Mori, S., and Reid, D. B., "Forty years of multiple hypothesis tracking - a review of key developments," in [2018 21st International Conference on Information Fusion (FUSION)], 452-459 (2018).

[14] Reid, D., "An algorithm for tracking multiple targets," IEEE Transactions on Automatic Control 24(6), 843-854 (1979).

[15] Kim, C., Li, F., Ciptadi, A., and Rehg, J. M., "Multiple hypothesis tracking revisited," in [2015 IEEE International Conference on Computer Vision (ICCV)], 4696-4704 (2015).

[16] Kalman, R., "A new approach to linear filtering and prediction problems," in [J. Basic Eng.], 35-45 (1960).

[17] Yang, S. and Baum, M., "Extended kalman filter for extended object tracking," in [2017 IEEE International Conference on Acoustics, Speech and Signal Processing (ICASSP)], 4386-4390 (2017).

[18] LeCun, Y., Bengio, Y., and Hinton, G., "Deep learning," in [Nature], 436-444 (2015).

[19] Albawi, S., Mohammed, T. A., and Al-Zawi, S., "Understanding of a convolutional neural network," in [2017 International Conference on Engineering and Technology (ICET)], 1-6 (2017).

[20] Chopra, S., Hadsell, R., and LeCun, Y., "Learning a similarity metric discriminatively, with application to face verification," in [2005 IEEE Computer Society Conference on Computer Vision and Pattern Recognition (CVPR'05)], 1, 539-546 vol. 1 (2005).

[21] Taigman, Y., Yang, M., Ranzato, M., and Wolf, L., "Deepface: Closing the gap to human-level performance in face verification," in [2014 IEEE Conference on Computer Vision and Pattern Recognition], 1701-1708 (2014).

[22] Wang, L., Xu, L., Kim, M. Y., Rigazico, L., and Yang, M.-H., "Online multiple object tracking via flow and convolutional features," in [2017 IEEE International Conference on Image Processing (ICIP)], 3630-3634 (2017).

[23] Zhou, H., Ouyang, W., Cheng, J., Wang, X., and Li, H., "Deep continuous conditional random fields with asymmetric inter-object constraints for online multi-object tracking," IEEE Transactions on Circuits and Systems for Video Technology 29(4), 1011-1022 (2019).

[24] Sadeghian, A., Alahi, A., and Savarese, S., "Tracking the untrackable: Learning to track multiple cues with long-term dependencies," in [2017 IEEE International Conference on Computer Vision (ICCV)], 300-311 (2017).

[25] Babaee, M., Li, Z., and Rigoll, G., "Occlusion handling in tracking multiple people using rnn," in [2018 25th IEEE International Conference on Image Processing (ICIP)], 2715-2719 (2018).

[26] Wieczorek, M., Rychalska, B., and Dabrowski, J., "On the unreasonable effectiveness of centroids in image retrieval," (2021). 
[27] Liao, S. and Shao, L., "Transformer-based deep image matching for generalizable person re-identification," (2021).

[28] Fu, D., Chen, D., Bao, J., Yang, H., Yuan, L., Zhang, L., Li, H., and Chen, D., "Unsupervised pre-training for person re-identification," (2021).

[29] Tang, S., Andriluka, M., Andres, B., and Schiele, B., "Multiple people tracking by lifted multicut and person re-identification," in [2017 IEEE Conference on Computer Vision and Pattern Recognition (CVPR)], 37013710 (2017).

[30] Ge, Y., Li, Z., Zhao, H., Yin, G., Yi, S., Wang, X., and Li, H., "Fd-gan: Pose-guided feature distilling gan for robust person re-identification," (2018).

[31] Li, Y.-J., Luo, Z., Weng, X., and Kitani, K. M., "Learning shape representations for clothing variations in person re-identification," (2020).

[32] Kim, C., Li, F., and Rehg, J. M., "Multi-object tracking with neural gating using bilinear lstm," in [ECCV], (2018).

[33] Chen, J., Sheng, H., Zhang, Y., and Xiong, Z., "A hybrid data association framework for robust online multi-object tracking," IEEE Transactions on Image Processing 26(12), 5667-5679 (2017).

[34] Ramachandra, K., [], CRC Press (2000).

[35] Coskun, H., Achilles, F., DiPietro, R., Navab, N., and Tombari, F., "Long short-term memory Kalman filters: Recurrent neural estimators for pose regularization," (2017).

[36] Zheng, T., Yao, Y., He, F., and Zhang, X., "An rnn-based learnable extended kalman filter design and application," in [2019 18th European Control Conference (ECC)], 3304-3309 (2019).

[37] Gordon, D., Farhadi, A., and Fox, D., "Re3 : Real-time recurrent regression networks for visual tracking of generic objects," (2018).

[38] He, K., Zhang, X., Ren, S., and Sun, J., "Deep residual learning for image recognition," (2015).

[39] Tan, M. and Le, Q. V., "Efficientnet: Rethinking model scaling for convolutional neural networks," (2020).

[40] Szegedy, C., Liu, W., Jia, Y., Sermanet, P., Reed, S., Anguelov, D., Erhan, D., Vanhoucke, V., and Rabinovich, A., "Going deeper with convolutions," (2014).

[41] Chen, T., Kornblith, S., Norouzi, M., and Hinton, G., "A simple framework for contrastive learning of visual representations," (2020).

[42] Mori, S., Chong, C.-Y., Tse, E., and Wishner, R., "Tracking and classifying multiple targets without a priori identification," IEEE Transactions on Automatic Control 31(5), 401-409 (1986).

[43] Roy, J., Duclos-Hindie, N., and Dessureault, D., "Efficient cluster management algorithm for multiplehypothesis tracking," in [Signal and Data Processing of Small Targets 1997], Drummond, O. E., ed., 3163, 301 - 313, International Society for Optics and Photonics, SPIE (1997).

[44] Blackman, S., "Multiple hypothesis tracking for multiple target tracking," IEEE Aerospace and Electronic Systems Magazine 19(1), 5-18 (2004).

[45] Danchick, R. and Newnam, G., "Reformulating reids mht method with generalised murty k-best ranked linear assignment algorithm," IEE Proceedings - Radar, Sonar and Navigation 153, 13-22(9) (February 2006).

[46] Brekke, E. and Chitre, M., "Success rates and posterior probabilities in multiple hypothesis tracking," in [2018 21st International Conference on Information Fusion (FUSION)], 949-956 (2018).

[47] Blackman, S. S., Dempster, R. J., and Reed, R. W., "Demonstration of multiple-hypothesis tracking (MHT) practical real-time implementation feasibility," in [Signal and Data Processing of Small Targets 2001], Drummond, O. E., ed., 4473, 470 - 475, International Society for Optics and Photonics, SPIE (2001).

[48] Papageorgiou, D. J. and Salpukas, M. R., "The maximum weight independent set problem for data association in multiple hypothesis tracking," in [Optimization and Cooperative Control Strategies], Hirsch, M. J., Commander, C. W., Pardalos, P. M., and Murphey, R., eds., 235-255, Springer Berlin Heidelberg, Berlin, Heidelberg (2009).

[49] Zheng, L., Shen, L., Tian, L., Wang, S., Wang, J., and Tian, Q., "Scalable person re-identification: A benchmark," in [Computer Vision, IEEE International Conference on], (2015). 
[50] Kristan, M., Matas, J., Leonardis, A., Felsberg, M., Cehovin, L., Fernandez, G., Vojir, T., Hager, G., Nebehay, G., Pflugfelder, R., Gupta, A., Bibi, A., Lukezic, A., Garcia-Martin, A., Saffari, A., Petrosino, A., and Solis Montero, A., "The visual object tracking vot2015 challenge results," in [2015 IEEE International Conference on Computer Vision Workshop (ICCVW)], 564-586 (2015). 